\title{
Patient Self-Collection of Group B Streptococcal Specimens During Pregnancy
}

\author{
Paul Hicks, MD, and Maria J. Diaz-Perez, MA
}

Objectives: In the United States, universal screening for group B streptococcal (GBS) colonization is recommended at 35 to 37 weeks' gestation. Previous studies have shown equivalent detection rates for GBS when women receive uniform instruction about specimen collection. It is unclear if these results would hold among patients with limited education given minimal, nonuniform instruction about collection technique.

Methods: A retrospective analysis of GBS culture results for physicians who practice universal patient self-collection of specimens were compared with GBS culture results for physicians who personally routinely collect the specimens at 2 sites within a community health center network. For self-collection, medical assistant staff provided minimal instruction to patients about collection technique and without a protocol. Patients in both groups were primarily Hispanic and of lower socioeconomic status.

Results: Patient self-collection occurred in 293 of 800 specimens $(36.6 \%)$. GBS was detected in $13.31 \%$ of patient self-collected samples and $10.65 \%$ of physician-collected specimens (relative risk, $1.25 ; 95 \% \mathrm{CI}, \mathbf{0 . 8 5}-1.84$ ). The study had $90 \%$ power to detect a $10 \%$ difference in colonization rates.

Conclusions: Patient self-collection with minimal instruction is not inferior to physician collection of specimens at detecting GBS colonization in a majority Hispanic population of lower socioeconomic status. (J Am Board Fam Med 2009;22:136-140.)

Group B streptococcal (GBS) colonization occurs in $10 \%$ to $30 \%$ of pregnancies. ${ }^{1,2}$ Invasive GBS affects 0.23 neonates per 1000 live births, with 4\% overall mortality. ${ }^{3}$

In 2002, the American College of Obstetricians and Gynecologists and the Centers for Disease Control and Prevention (CDC) recommended universal vaginal-rectal screening for GBS of women at 35 to 37 weeks' gestation. ${ }^{4,5} \mathrm{~A}$ variety of approaches have been proposed, including vaginalperineal specimen collection instead of vaginal-rec$\mathrm{tal}^{6}$ and culture or rapid antigen testing at the time of delivery. ${ }^{7,8}$ The unifying feature among the usual collection methods is that the health care professional obtains the specimen. However, for other conditions, including sexually transmitted

This article was externally peer reviewed.

Submitted 15 January 2008; revised 23 September 2008; accepted 23 September 2008.

From the Salud Family Health Centers, Fort Lupton, Colorado.

Funding: none.

Prior presentation: This study was presented at the North American Primary Care Research Group Annual Meeting, October 20-24, 2007, Vancouver, Canada.

Conflict of interest: none declared.

Corresponding author: Paul Hicks, MD, Salud Family Health Centers, 203 South Rollie Street, Fort Lupton, CO 80621 (E-mail: phicks@uph.org). diseases, it has been shown that patient self-collection of specimens is as sensitive as physician collection. Furthermore, this diagnostic equivalency has been noted across demographic groups at varying socioeconomic levels and is, in fact, preferred to physician collection of specimens by many patients. $^{9-11}$

Similar results have been obtained with GBS specimen collection. ${ }^{12-15}$ Earlier studies were limited by smaller sample size and collection of specimens at gestational ages not consistent with recommendations. Most recently, Price et $\mathrm{al}^{16}$ published the largest study to date that assessed self-collection with 330 participants in a Canadian maternity center. They compared self-collection to physician collection and assessed patient preference of collection method. They found that after standardized and uniform instructions, women who collected their specimens themselves had detection rates equivalent to those women whose physicians collected their specimens. More than half of women preferred self-collection to physician collection at the end of the study. Ethnicity and socioeconomic status of the patients were not assessed.

The questions that remain are whether equivalency in detection rates would hold (1) in usual 
practice, outside of a research setting; (2) without structured or uniform patient education; and (3) among patients who may have a preference against self-collection.

\section{Methods}

Salud Family Health Centers is a large community health center network in Northeastern Colorado. Seventy-five percent of the patients are Hispanic and $85 \%$ are below $200 \%$ of the federal poverty level (in 2005, the United States federal poverty level was an income of $\leq \$ 19,350$ per annum for a family of 4). Previous work at our institution showed a fourth-grade average reading level (personal communication, Tillman Farley, 2007).

In our institution, physician care relating to GBS collection can be divided into 3 groups: (1) physicians who always collect the specimens themselves, (2) physicians who offer their patients a choice between self-collection and physician collection, and (3) physicians who practice universal patient self-collection and do not allow for physician collection of swabs. This latter group instructs their medical assistants to direct patients to selfcollect their specimens, treating collection of GBS as qualitatively equivalent to patient self-collection of urine specimens.

Two sites in the network were included for study. They were chosen because they have physicians who practice universal patient self-collection of GBS specimens and have other physicians who only permit physician collection who could serve as controls.

Patients are assigned in rotation to family physicians or physician extenders. Patients can opt out of their assignment and choose a particular provider. Physician extenders are teamed with specific physicians and patient visits alternate between the 2 providers. At approximately 32 to 34 weeks' gestation, care is transferred entirely to the physician. Physicians also form teams among themselves to enhance familiarity of the patients with the active physician pool at the site and, hopefully, to maximize comfort and satisfaction at time of delivery; and to allow coverage for physicians when they are out of the office.

Group B streptococcus screening follows American College of Obstetricians and Gynecologists and CDC recommendations of a single vaginalrectal swab at 35 to 37 weeks' gestation cultured on a selective media by an outside commercial laboratory service. For those physicians who practice universal patient self-collection, there are no standardized instruction materials or script that is followed by the medical assistant, who gave directions in Spanish or English according to the language preference of the patient. In one of 4 bathrooms, a poster showed how to collect the specimen. Patient preference for self-collection was not assessed and patients were not offered the option of physiciancollection of specimens.

All 1022 GBS tests performed at the 2 sites in 2005 were initially considered and sorted by the provider who ordered the specimen. To ensure the accuracy of the data, chart review was performed for those specimens for which the provider of record was not indicated, as well as for a subset of clearly identified specimens. Results for providers who were inconsistent in their collection method (ie, who allowed the patient to chose self-collection or provider collection) were excluded because the method of collection could not be discerned from a review of the chart or the laboratory data, leaving a final sample of 800 results.

Demographic information was not collected because few, if any, significant demographic variables affect GBS colonization rates. ${ }^{17,18}$ Although Stapleton et $\mathrm{a}^{18}$ found a 1.54 relative risk of colonization among African-American women, this subgroup historically makes up less than $1 \%$ of our prenatal population.

Power was calculated in a post hoc manner with SAS statistical software (version 9.1, SAS Institute, Inc., Cary, NC) using Pearson $\chi^{2}$ statistics. Power curves were generated for each site and the total sample under the following assumptions: (1) percentage of GBS-positive patients among self-testers will be lower than the percentage of GBS-positive patients with usual care; (2) using the most conservative baseline rate of colonization, that is $30 \%$ of GBS-positive patients in self-testers, the effect size (ie, $0 \%$ to $20 \%$ proportional difference) was taken as the increasing difference from this percent; (3) $\alpha$ level of significance of 0.05 ; and (4) 1-tailed test (because we are interested in a directional difference).

$\chi^{2}$ analysis and relative risks were calculated using Epi Info (version 6.0, Centers for Disease Control and Prevention, Atlanta, GA). The Migrant Clinicians Network Institutional Review Board evaluated the project and found it to be exempt. 
Table 1. Rates of Group B Streptococcal Detection: Patient-Collected Vs Provider-Collected Specimens

\begin{tabular}{|c|c|c|c|c|c|c|}
\hline & Relative Risk (95\% CI) & $\chi^{2}$ & $P$ & Patient Self-Collection & Usual Care & Totals \\
\hline Site 1 & $1.49(0.85-2.63)$ & 1.94 & .16 & & & \\
\hline GBS+ & & & & 21 & 22 & 43 \\
\hline GBS- & & & & 144 & 236 & 380 \\
\hline Totals & & & & 165 & 258 & 423 \\
\hline Rate of positive tests & & & & $12.73 \%$ & $8.53 \%$ & \\
\hline Site 2 & $1.11(0.64-1.87)$ & 0.11 & .74 & & & \\
\hline GBS+ & & & & 18 & 32 & 50 \\
\hline GBS- & & & & 110 & 217 & 327 \\
\hline Totals & & & & 128 & 249 & 377 \\
\hline Rate of positive tests & & & & $14.06 \%$ & $12.85 \%$ & \\
\hline Overall & $1.25(0.85-1.84)$ & 1.28 & .26 & & & \\
\hline GBS+ & & & & 39 & 54 & 93 \\
\hline GBS- & & & & 254 & 453 & 707 \\
\hline Totals & & & & 293 & 507 & 800 \\
\hline Rate of positive tests & & & & $13.31 \%$ & $10.65 \%$ & \\
\hline
\end{tabular}

GBS+, group B streptococcal positive; GBS-, group B streptococcal negative.

\section{Results}

A total of 800 GBS specimens were considered for analyses, with 423 from site 1 and 377 from site 2 (see Table 1). GBS-positive cultures occurred in $10.17 \%$ of the swabs taken from site 1 and $13.26 \%$ of cultures from site 2, with an overall GBS colonization rate of $11.63 \%$. Patient self-collection occurred among $39 \%$ of all patients in site $1,33.95 \%$ at site 2 , and $36.63 \%$ overall.

Across the 2 sites, the rate of positive cultures in the self-collection group was not significantly different to the rate of positive culture in the usual care group. For the whole sample, the rate of positive GBS was $13.31 \%$ in the self-collection group compared with $10.65 \%$ in the physician-collection group (relative risk, 1.25; 95\% CI, 0.85-1.84) (see Table 1). Considering the total sample, the study had $90 \%$ power to detect a $10 \%$ difference in rates of GBS colonization. Considering the individual clinics, the sample sizes are sufficient at $80 \%$ power to detect less than a $15 \%$ difference.

\section{Comment}

Compared with physician-collected specimens, the rate of detection of GBS colonization for patient self-collection was the same in this population of majority Hispanic women of lower socioeconomic status. Although the findings are consistent with earlier studies about the self-collection of GBS, 2 points of disparity with regard to the collection process compared with previous work are notable.
First, there was nonuniform patient instruction about collection technique. Second, no adjustment to care was made to account for possible patient preferences against performing self-collection of GBS specimens, which could lead to poor specimen collection and lower rates of detection.

The major strength of this study is that it brings the literature closer to a typical practice setting. Control and uniformity of educational interventions maximize the internal validity in research. There is sometimes difficulty, though, in applying those interventions in practice given the wide variations seen day-to-day in practices in the United States and the inconsistencies in adherence to protocol-based interventions. We feel that this study, with its nonuniform instruction in a minority population with lower health literacy and socioeconomic status, goes a long way in reinforcing previous works as to the equivalence of these 2 collection methods. Furthermore, this study adds to the growing body of literature that addresses health care needs and disparities among vulnerable populations.

There are several limitations that deserve discussion. First, there was no comparison to a gold standard. Previous studies were designed prospectively and included a contemporaneous specimen collected on that same patient by the medical provider. This study, however, was designed as a retrospective analysis of usual care. Our objective was to determine whether the practice of self-collection 
was inferior. Given the large sample size and the high degree of statistical power, we believe we have answered that question. Although the conclusions may be made with more surety in a prospective, randomized study, we feel also that our study design and sample size were sufficient to support the conclusions.

A second limitation is one of potential selection bias. Because patients were not randomized to providers, it is possible that those cared for by the physicians who practice self-collection have substantially higher rates of GBS colonization than patients of other physicians such that similar rates of detection would actually show self-collection to be inferior.

In considering this, although it is true that they were not randomized, selection bias implies consistent allocation to the group. Given our practice model requiring physicians to participate in the hospitalist pool and team coverage within the clinic between nurse practitioner/physician assistant and physicians in early pregnancy and between physicians in the last trimester, we believe that the possibility of maintaining a rigid cohort of high-risk patients at the end of their pregnancies is significantly attenuated.

Lastly, this study is notable for not inquiring about patient preferences about the method of testing. Although this may represent a limitation of the health care system in place at those sites, it is not clear that it weakens this study or its conclusions. On the contrary, assuming that $30 \%$ to $40 \%$ of patients would prefer not to self-collect, as seen in the above studies, that potential preference to the contrary did not manifest itself in lower rates of detection in our study. This suggests a population very motivated toward the health of their babies who collect excellent specimens despite potentially preferring not to. This opens the door to selfcollection in other settings, such as the collection of swabs at home before the visit if the patient preferred or if analysis showed that to be a time savings for the patient or provider during the visit. It is certainly the case that some patients do prefer this route of collection and, although not assessed in this study, the allowance of this practice may improve patient satisfaction.

From this study we conclude that patient selfcollection of GBS specimens is not inferior in detecting GBS colonization compared with provider collection of swabs among a majority of Hispanic women of lower socioeconomic status at 35 to 37 weeks' gestation in our health center. Patient education about swab collection methods need not be standardized or lengthy.

We would like to thank Betty Metz for her assistance with data collection and her commitment to this project, and Caroline Esserman for her input on statistical methods.

\section{References}

1. Hammerschlag MR, Baker CJ, Alpert S, et al. Colonization with group B streptococci in girls under 16 years of age. Pediatrics 1977;60:473-6.

2. Regan JA, Klebanoff MA, Nugent RP. Vaginal infections and prematurity study group. The epidemiology of group B streptococcal colonization in pregnancy. Obstet Gynecol 1991;77:604-10.

3. Schrag SJ, Zywicki S, Farley MM, et al. Group B streptococcal disease in the era of intrapartum antibiotic prophylaxis. N Engl J Med 2000;342:15-20.

4. American College of Obstetricians and Gynecologists. ACOG Committee Opinion: number 279, December 2002. Prevention of early-onset group B streptococcal disease in newborns. Obstet Gynecol 2002;100:1405-12.

5. Schrag S, Gorwitz R, Fultz-Butts K, Schuchat A. Prevention of perinatal group B streptococcal disease. Revised guidelines from CDC. MMWR Recomm Rep 2002;51(RR-11):1-22.

6. Jamie WE, Edwards RK, Duff P. Vaginal-perianal compared with vaginal-rectal cultures for identification of group B streptococci. Obstet Gynecol 2004; 104(5 pt 1):1058-61.

7. Ryan KM, Lencki SG, Elder BL, Northern WI, Khamis HJ, Bofill JA. DNA probe for beta-hemolytic group B streptococcus. Diagnostic accuracy in threatened preterm labor. J Reprod Med 1999;44: 587-91.

8. Yancey MK, Armer T, Clark P, Duff P. Assessment of rapid identification tests for genital carriage of group B streptococci. Obstet Gynecol 1992;80:1038-47.

9. Garland SM, Tabrizi SN. Diagnosis of sexually transmitted infections (STI) using self-collected non-invasive specimens. Sex Health 2004;1:121-6.

10. Richardson E, Sellors JW, Mackinnon S, et al. Prevalence of Chlamydia trachomatis infections and specimen collection preference among women, using self-collected vaginal swabs in community settings. Sex Transm Dis 2003;30:880-5.

11. Holland-Hall CM, Wiesenfeld HC, Murray PJ. Self-collected vaginal swabs for the detection of multiple sexually transmitted infections in adolescent girls. J Pediatr Adolesc Gynecol 2002;15:307-13.

12. Mercer BM, Taylor MC, Fricke JL, Baselski VS, Sibai BM. The accuracy and patient preference for 
self-collected group B streptococcus cultures. Am J Obstet Gynecol 1995;173:1325-8.

13. Spieker MR, White DG, Quist BK. Self-collection of group B streptococcus cultures in pregnant women. Mil Med 1999;164:471-4.

14. Torok PG, Dunn JR. Self-collection of antepartum anogenital group B streptococcus cultures. J Am Board Fam Pract 2000;13:107-10.

15. Molnar P, Biringer A, McGeer A, McIsaac W. Can pregnant women obtain their own specimens for group B streptococcus? A comparison of maternal versus physician screening. The Mount Sinai GBS Screening Group. Fam Pract 1997;14:403-6.
16. Price D, Shaw E, Howard M, Zazulak J, Waters H, Kaczorowski J. Self-sampling for group B streptococcus in women 35 to 37 weeks pregnant is accurate and acceptable: a randomized cross-over trial. J Obstet Gynaecol Can 2006;28:1083-8.

17. Terry RR, Kelly FW, Gauzer C, Jeitler M. Risk factors for maternal colonization for group $\mathrm{G}$ betahemolytic streptococci. J Am Osteopath Assoc 1999; 99:571-3.

18. Stapleton RD, Kahn JM, Evans LE, Crichlow CW, Gardella CM. Risk factors for group B streptococcal genitourinary tract colonization in pregnant women. Obstet Gynecol 2005;106:1246-52. 